\title{
Combined Laminoplasty and Posterior Fusion for Cervical Spondylotic Myelopathy Treatment: A Literature Review
}

\author{
Kelly J. Bridges ${ }^{*}$ Lauren N. Simpson ${ }^{*}$, Carli L. Bullis, Andy Rekito, Christina M. Sayama, Khoi D. Than \\ Department of Neurological Surgery, Oregon Health \& Science University, Portland, OR, USA
}

\begin{abstract}
Study Design: A literature review.
Purpose: To explore the utility of laminoplasty in combination with instrumented fusion, with a focus on neurological outcomes and changes in kyphotic deformity.

Overview of Literature: Management of cervical spondylotic myelopathy (CSM) to reduce morbidity within the neurosurgical population.

Methods: A US National Library of Medicine PubMed search was conducted for manuscripts pertaining to cervical laminoplasty and fusion for the management of CSM. Several relevant studies were shortlisted for review, and the bibliographies of the articles were searched for additional references. The search was limited to human studies, English-language literature, and reports on more than one patient.

Results: Combined laminoplasty and fusion was found to provide at least comparable, if not superior, neurological outcomes in specific patient populations with CSM. The Japanese Orthopedic Association scores, local kyphosis, and C2-C7 angle have been reviewed in several manuscripts, and improvement in each of these categories was found with laminoplasty and fusion.

Conclusions: The treatment of CSM necessitates an individualized approach based on the pathoanatomical variation. Laminoplasty and fusion can be appropriately used for patients with CSM in a setting of local kyphotic deformity, ossification of the posterior longitudinal ligament, associated segmental instability, and the need for strong stabilization.
\end{abstract}

Keywords: Cervical spondylosis; Laminoplasty; ossification; Kyphosis

\section{Introduction}

Optimizing the management of cervical spondylotic myelopathy (CSM) has a significant potential in reducing morbidity within the neurosurgical population. CSM is a common cause of hospital admissions as well as the leading cause of spinal cord dysfunction in patients aged $>55$ years [1]. Moreover, CSM is a life-long degenerative process that is accompanied by non-inflammatory disk degeneration, facet joint osteoarthritis, and posterior longitudinal ligament and ligamentum flavum hypertrophy, laxity, and ossification. It often occurs in individuals

Received Apr 11, 2017; Revised Sep 8, 2017; Accepted Oct 2, 2017

Corresponding author: Khoi D. Than

Department of Neurological Surgery, Oregon Health \& Science University, Mail Code CH8N, 3303 SW Bond Avenue, Portland, Oregon 97239

Tel: +1-503-494-4314, Fax: +1-503-346-6810, E-mail: thank@ohsu.edu

${ }^{*}$ These authors contributed equally to this work. 
with congenitally narrowed spinal canals [2]. Static forces such as decreased canal diameter, dynamic forces such as repeated compressive trauma, and hypoperfusion injury due to cord ischemia alter the pathological cervical cord, which manifests as CSM [3]. While a minority of patients with mild CSM can be managed conservatively with neck immobilization, traction, and physical therapy, most patients with CSM require surgical intervention to prevent further worsening of their neurological functions [4]. For patients with moderate to severe CSM, a neurological decline occurs in a stepwise fashion in $75 \%$ of the cases, slowly and steadily in $20 \%$ of the cases, and rapidly in $5 \%$ of the cases [2].

The operative management for patients with CSM aims to decompress the spinal cord, restore sagittal alignment, and stabilize the spine. There are various surgical approaches that can accomplish these goals. A decision regarding the optimal surgical approach should take the following factors into consideration: (1) sagittal curvature, (2) locations of the compressive pathology, (3) number of levels involved, and (4) patient age. Anterior decompression with graft placement and fusion is traditionally preferred when the cervical spine is straightened or effectively kyphotic as well as when there are less than three levels of compression. In addition, the presence of concomitant cervical radiculopathy and anterior midline and/or paramedian compressive pathology may be better addressed via an anterior approach. Posterior decompression may be preferable when cervical lordosis is effectively maintained, when there are more than three levels of compression, and when posterior ligamentous hypertrophy and/or ossification contributes most to the compressive pathology. Even when ventral pathology is the predominant contributor to cord compression in the lordotic spine, a posterior surgical approach can indirectly decompress by allowing for dorsal migration of the spinal cord away from the pathology [3].

Ultimately, pathoanatomical variations noted in patients with CSM necessitate an individualized approach for determining a surgical intervention that will optimize effectiveness and safety $[5,6]$. One option that has been less studied over others is the combination of posterior cervical fusion and laminoplasty (Fig. 1). The goal of this type of procedure is to decompress the spinal cord while providing solid fixation and ample bony surface to accomplish successful fusion. The purpose of this literature review was to explore the utility of laminoplasty in com-

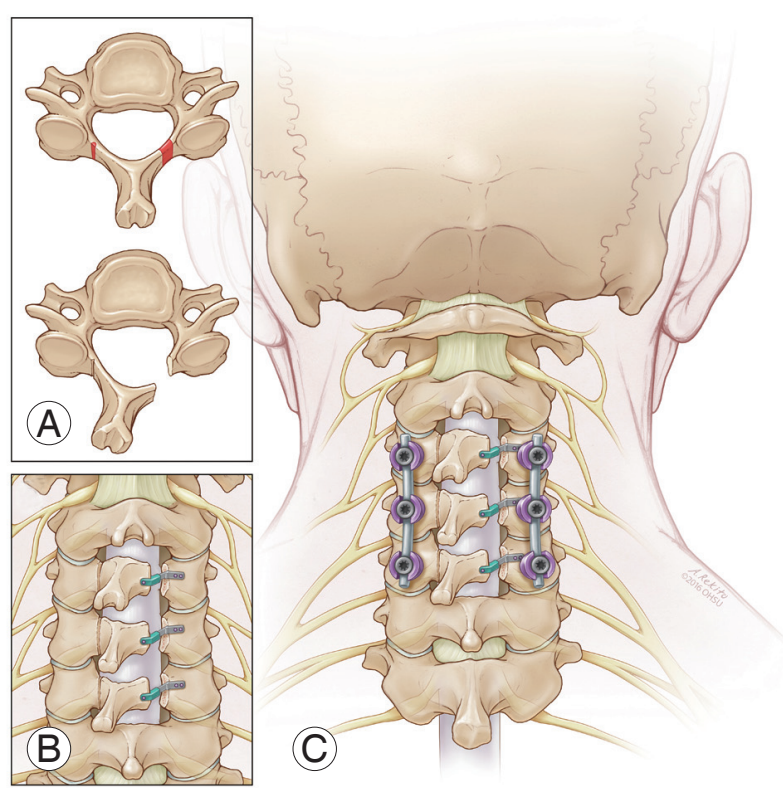

Fig. 1. Illustration of the combination of a posterior instrumented cervical fusion with laminoplasty. (A) To perform the laminoplasty, a trough is drilled at the junction of the lamina and lateral mass while preserving the inner cortex, which acts as a hinge. A hemilaminotomy is performed on the contralateral side, which allows decompression of the spinal cord while preserving several of the dorsal elements of the spine. (B) The spinal canal is well decompressed, and the lamina is repositioned with appropriate hardware such as titanium miniplates. (C) Lateral mass screws and rods are placed bilaterally in combination with the unilateral laminoplasty hardware.

bination with instrumented fusion, looking specifically at neurological outcomes and changes in kyphotic deformity.

\section{Materials and Methods}

\section{Information source}

A US National Library of Medicine PubMed search was conducted for articles pertaining to cervical laminoplasty and fusion for the management of CSM. A PICOS-P (problem/patient/population), I (intervention/indicator), C (comparison), O (outcome), and S (setting) - worksheet and search strategy was created and followed (data not shown). Institutional review board approval was not required for a literature review.

\section{Search}

The initial search items used included "cervical," "laminoplasty," "fusion," and "myelopathy." Article reference lists and suggested similar studies through PubMed were 

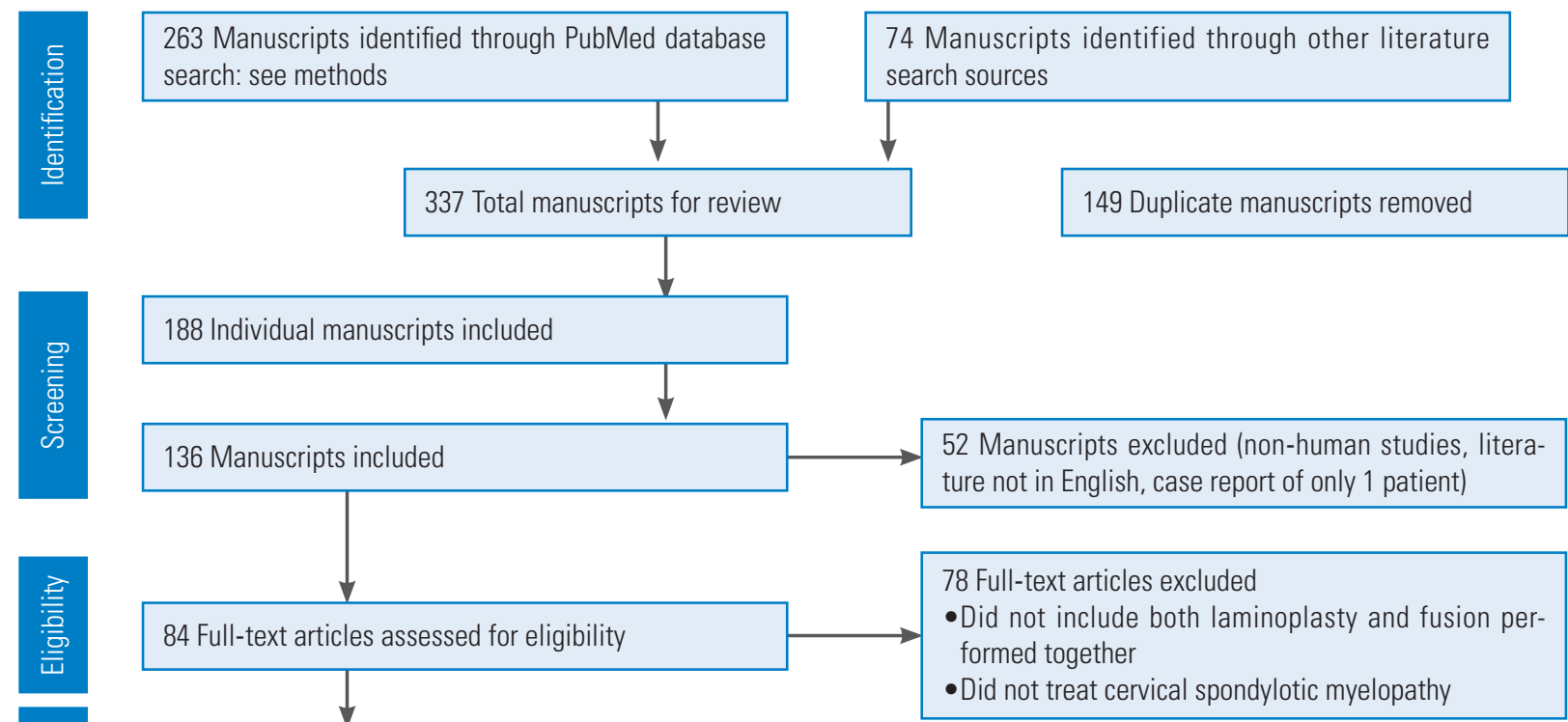

6 Studies included in qualitative and quantitative synthesis

Fig. 2. PRISMA (preferred reporting items for systematic reviews and meta-analyses) flow diagram for the selection of studies based on the inclusion criteria during a literature review (http://www.prisma-statement.org/).

checked to identify additional pertinent studies. The search was limited to human studies, English-language literature, and reports on more than one patient. A total of 263 articles were initially identified (Fig. 2). A PRISMA (preferred reporting items for systematic reviews and meta-analyses), flow chart (an evidence-based minimum set of items for reporting in systematic reviews and metaanalyses) was then utilized (Fig. 2).

\section{Selection}

The criteria for final selection led by two of the authors (KJB and LNS) included patients with myelopathy, in which both cervical laminoplasty and fusion were performed together. Based on these criteria, six articles were included in the quantitative analysis, all of which were retrospective case series reviewing patient data between 1995 and 2009 (Fig. 2).

\section{Data collection process and items}

The principle summary measures included the Frankel grade (FG) of spinal cord injury; the Barthel Index, which measures functional status by assessing activities of daily living; the Japanese Orthopedic Association (JOA) Score to assess the clinical symptoms in a setting of CSM; the
Visual Analog Scale for rating pain; and the $\mathrm{C} 2-\mathrm{C} 7$ angle to assess kyphosis. Data from each study were reviewed and placed in a table format to allow for comparative assessment of all records (Table 1) [7-12].

\section{Results}

Pediatric neurosurgeons have been using combined laminoplasty and non-instrumented posterolateral fusion for the treatment of spinal cord tumors and other spinal pathological process since at least 1981 [13]. In children and adolescents, laminoplasty and posterolateral fusion have been performed to avoid post-laminectomy kyphotic deformity due to extensive posterior element destruction in the developing spine [13]. Shikata et al. [13] compared laminectomy and fusion with laminoplasty and fusion in a pediatric population and found lesser blood loss and no post-procedure spinal deformity or instability with the use of laminoplasty and fusion. Pediatric spine stability depends on the proper balance of all posterior elements until after the growth spurt. As such, the utility of this surgical approach was not generalized to adults, wherein the adult spine stability primarily depends on the facet joints among its posterior elements [14]. 


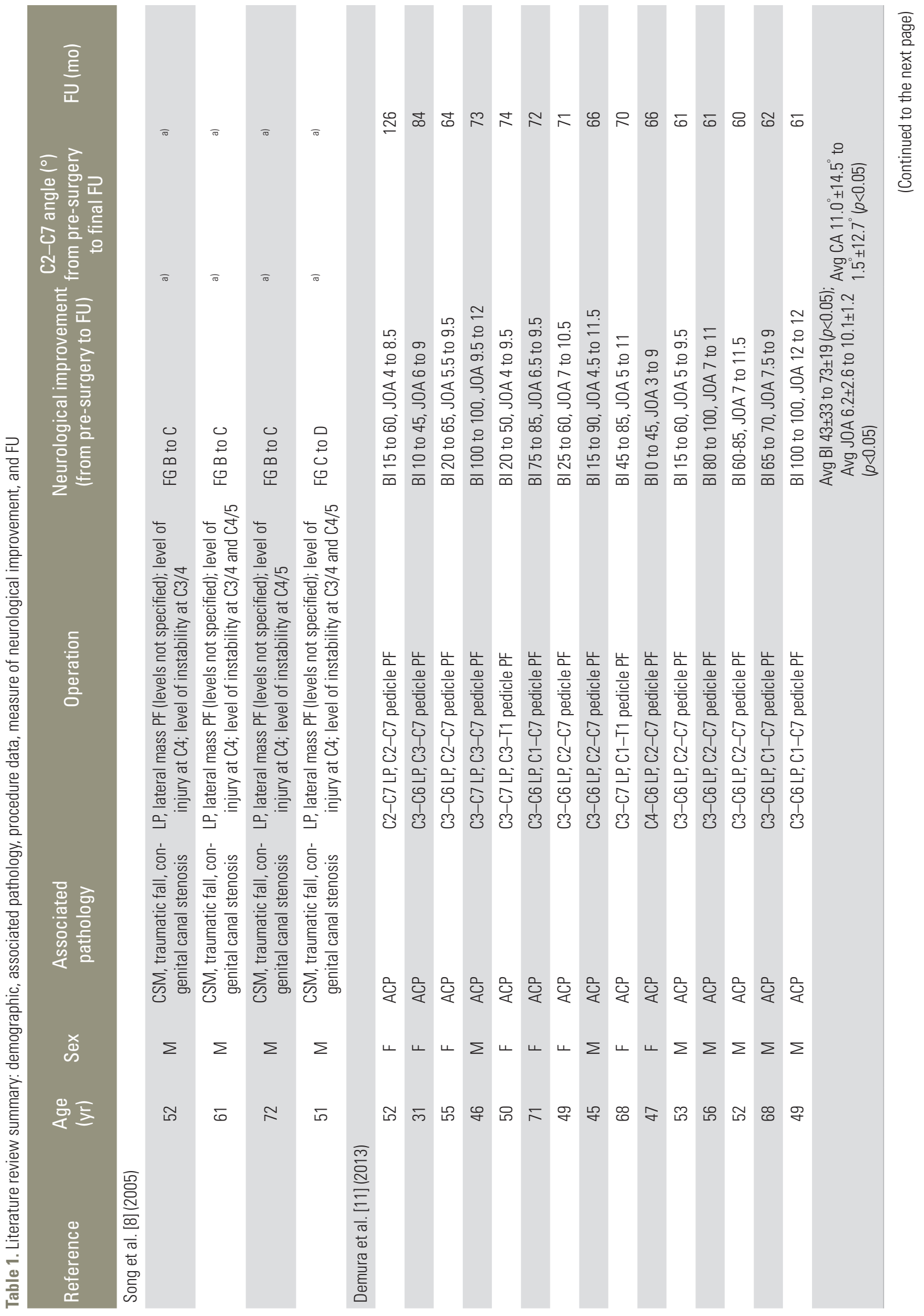




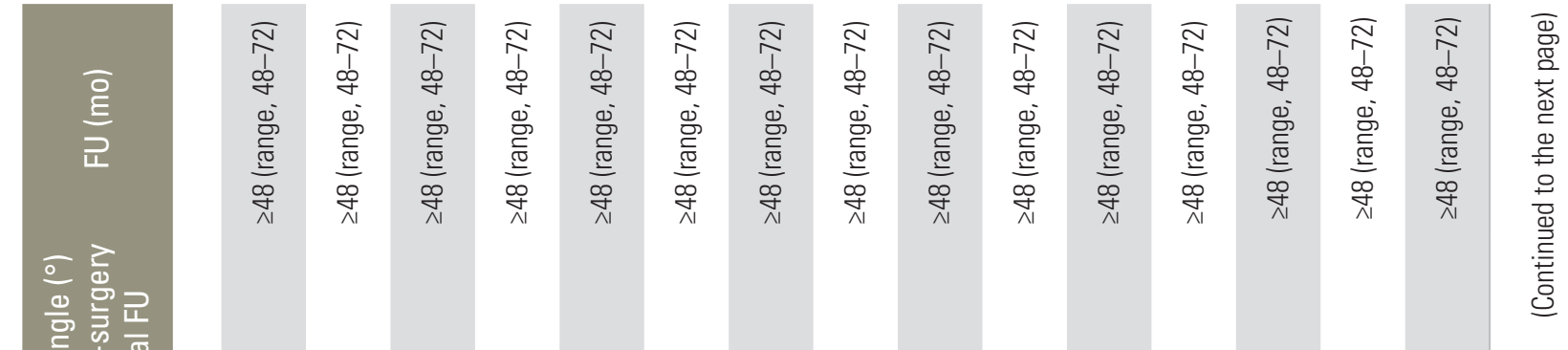

可高点

ปิ

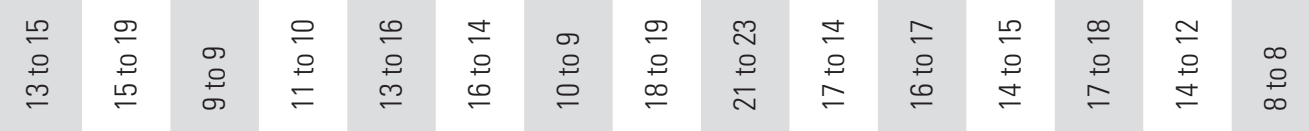

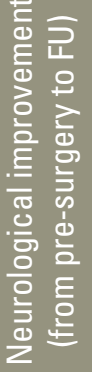

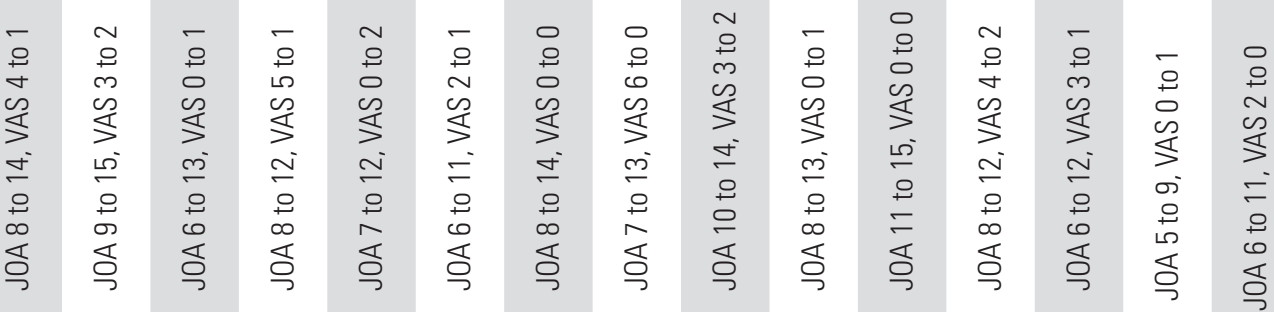

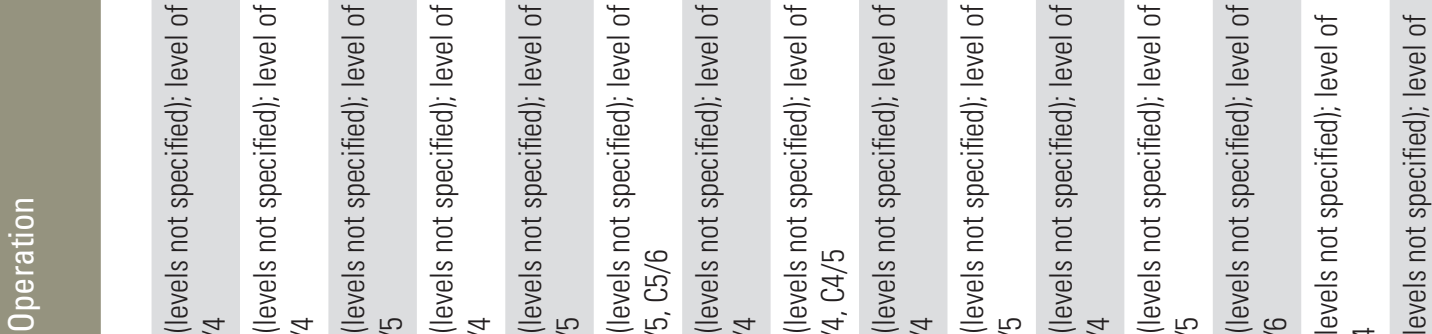
产嵌

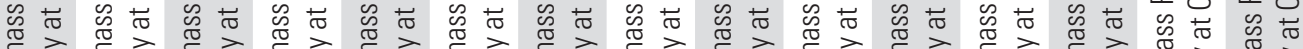

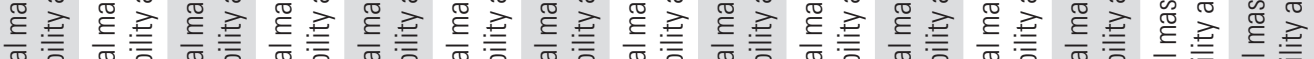

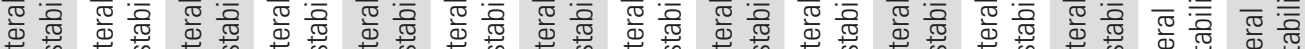

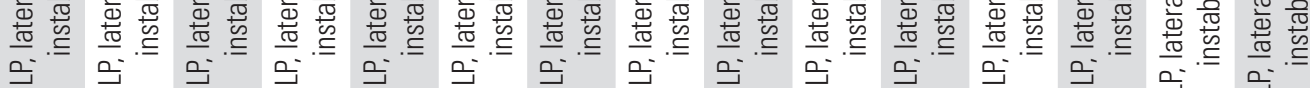

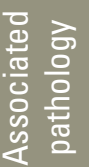

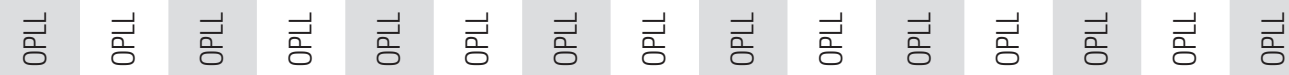
ळ

$\stackrel{\infty}{\frac{0}{4}}$

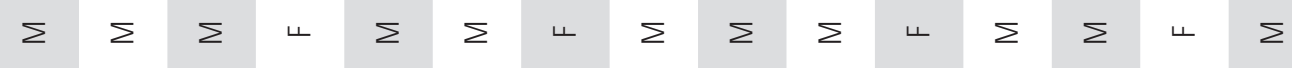

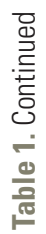

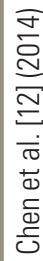
范
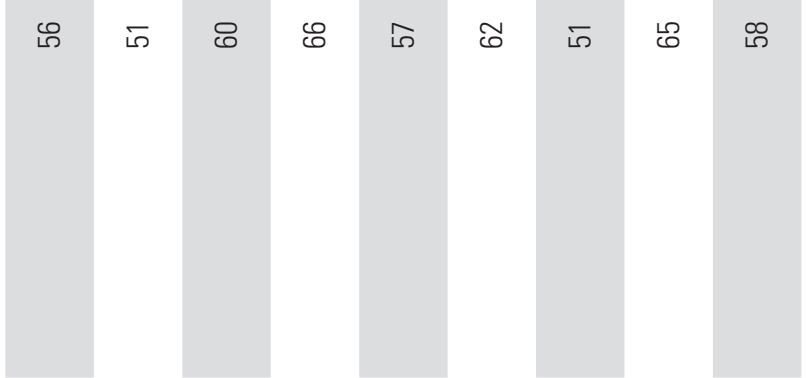


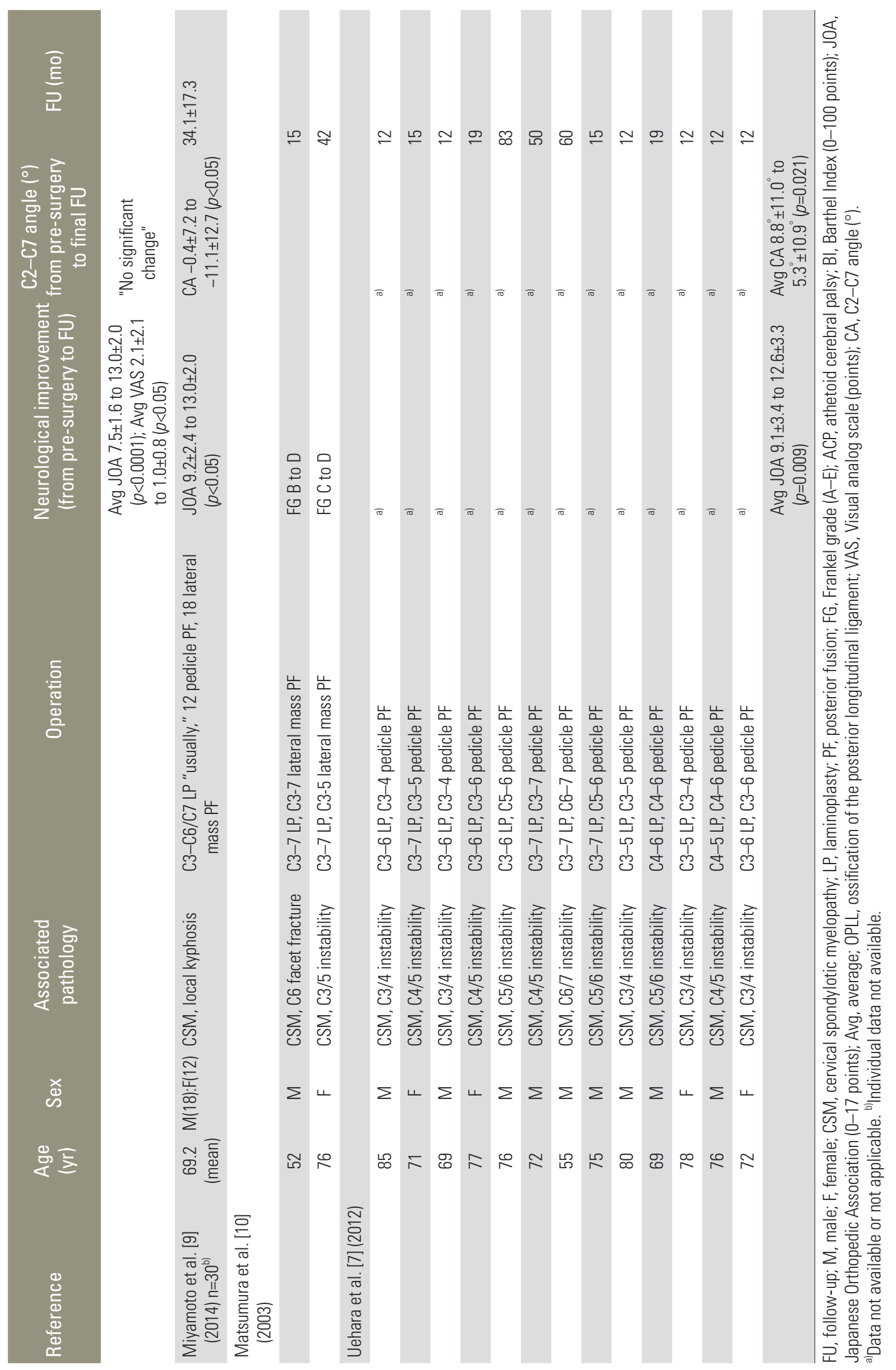




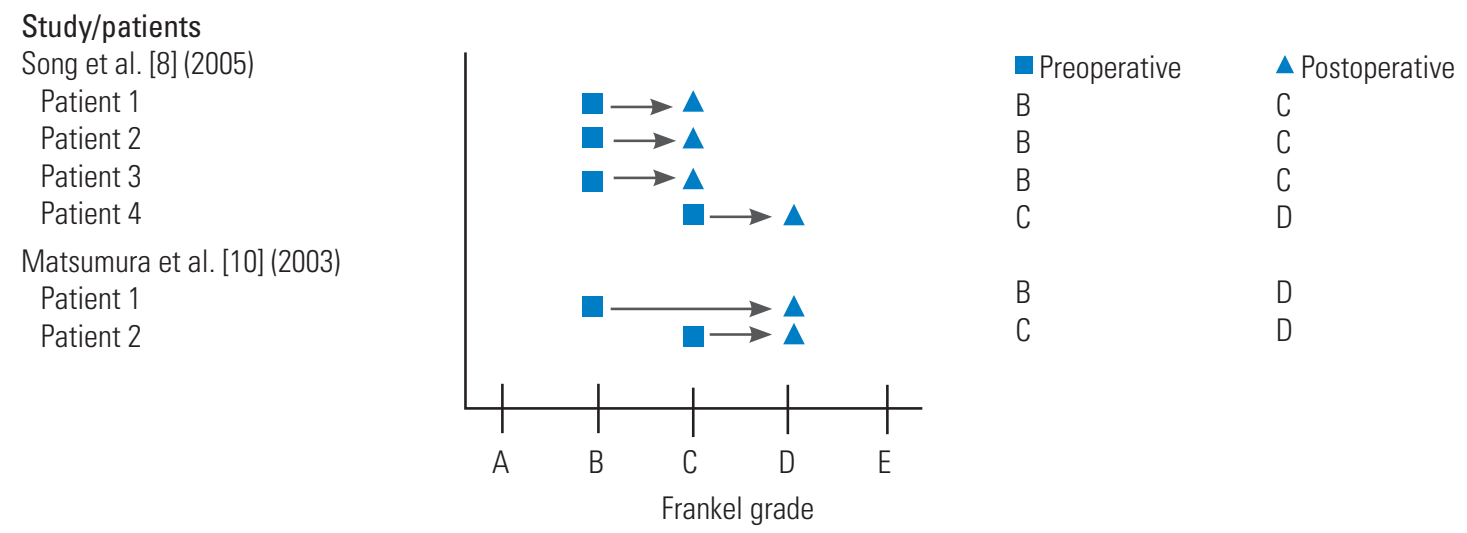

Fig. 3. A forest plot of preoperative and postoperative Frankel grades when reported.

\section{Cervical spondylotic myelopathy and instability}

In 2003, Matsumura et al. [10] described a case series of two adult patients with multilevel CSM and spinal instability, who were treated with posterior stabilization using lateral mass plates and expanding laminoplasty by using interspinous iliac bone graft. One of these patients was a 76-year-old woman who presented with progressive motor weakness and sensory disturbances in both upper and lower extremities [10]. Her multilevel CSM was due to a kyphotic deformity with instability from $\mathrm{C} 3$ to $\mathrm{C} 5$, as demonstrated by significant motion on dynamic cervical radiographs. Magnetic resonance imaging (MRI) revealed degenerative spinal canal stenosis and spinal cord compression. The patient underwent laminoplasty with interspinous iliac bone graft and posterolateral mass plating from C3 to C5. The surgeons bent the lateral mass plates in the procedure to accommodate her cervical lordosis. Her deficits had improved by the time of discharge and she could perform her daily activities without any support by 3-week follow-up visit (Fig. 3). Complete bony fusion was radiographically evident at 15 months postoperatively. Three and a half years after her operation, she remained independent with no radiographic evidence of instability or re-stenosis.

In 2012, Uehara et al. [7] retrospectively reviewed 13 patients who had CSM and instability from 1998 to 2010, specifically excluding patients with trauma, rheumatoid arthritis, destructive spondyloarthropathy, spinal tumors, or athetoid cerebral palsy. Instability was defined by the White and Panjabi's guidelines for radiographic instability, including vertebral body slip length of $>3.5 \mathrm{~mm}$ and a vertebral body angle change of $>11^{\circ}$ between the adjacent vertebral bodies. However, if the patients' symptoms were severe and spinal cord compression was mild, they underwent posterior instrumented fusion regardless of whether the radiographic measurements met the above criteria. Cervical pedicle screws were placed using a computed tomography-based navigation system, after which expansive open-door laminoplasty was performed. The JOA scoring system, which measures the motor and sensory functions, was used to evaluate CSM before surgery, immediately after surgery, and at the final follow-up, ranging from 12 to 83 months. The cervical sagittal alignment (C2$\mathrm{C} 7$ lordotic angle) was compared at similar time periods. The results revealed that the JOA scores had significantly improved from $9.1 \pm 3.4$ before surgery to $12.6 \pm 3.3$ at the final follow-up ( $p=0.009$ ) (Fig. 4). The average C2-C7 lordotic angle was $8.8^{\circ} \pm 11.0^{\circ}$ preoperatively and $5.3^{\circ} \pm 10.9^{\circ}$ $(p=0.021)$ at the final follow-up (Fig. 5).

Miyamoto et al. [9] retrospectively reviewed 60 patients with CSM and kyphotic deformity who had undergone either laminoplasty alone or laminoplasty with posterior fusion at three local centers from 1999 to 2009. Thirty additional patents with CSM, but no kyphotic deformity, were used as controls and they underwent laminoplasty alone. As reported by Matsumura et al. [10], Kurokawa's lamina splitting method was used for these patients, but the hydroxyapatite spacers were placed between the lamina, as opposed to that in the bone graft. Of the $30 \mathrm{pa}-$ tients who underwent combined laminoplasty and fusion, 18 received lateral mass screws and 12 received pedicle screws. The primary outcomes were the recovery rate of the JOA score, local kyphosis, and $\mathrm{C} 2-\mathrm{C} 7$ angle. The authors found that the recovery rate of the JOA score was significantly worse in the laminoplasty-only patients than in the laminoplasty and fusion patients $(32.6 \%$ versus $44.5 \%, p<0.05)$. They a significant difference in the correc- 


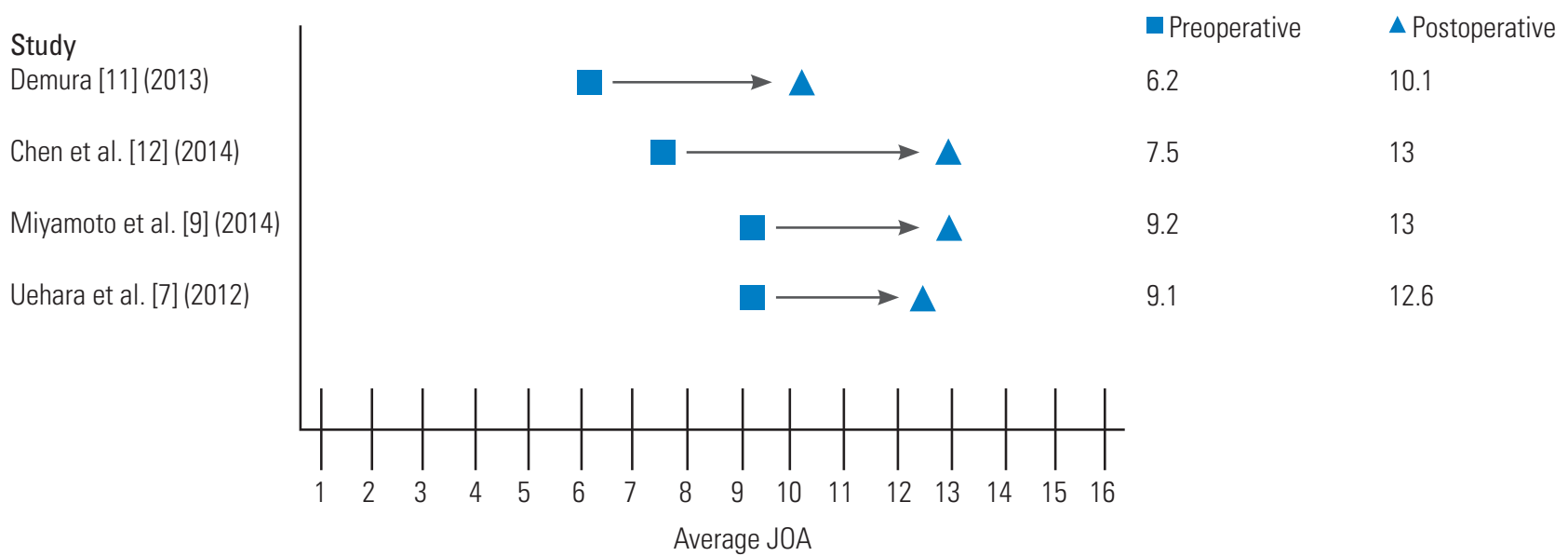

Fig. 4. A forest plot of average preoperative and postoperative JOA scores when reported. JOA, Japanese Orthopedic Association.

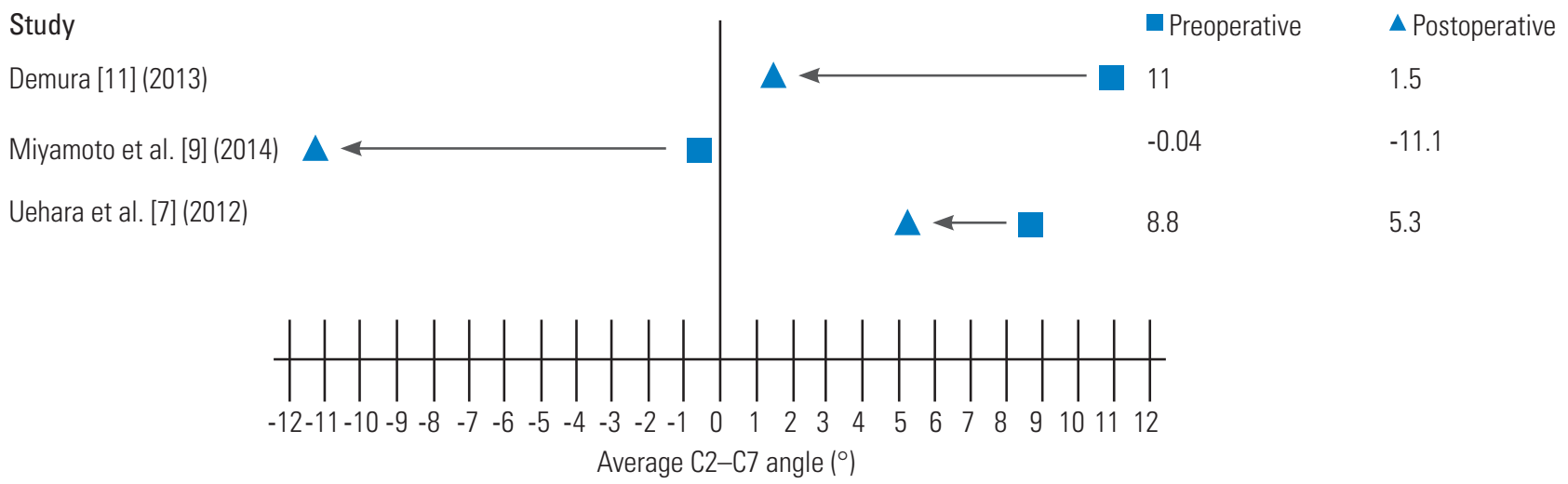

Fig. 5. A forest plot of average preoperative and postoperative C2-C7 angles when reported.

tion of local kyphotic angle with a mean correction angle of $11.1^{\circ}$ postoperatively in the laminoplasty and fusion group, whereas the laminoplasty-only group maintained a local kyphotic angle $(p<0.05)$. The $\mathrm{C} 2-\mathrm{C} 7$ angle was improved at the follow-up for the laminoplasty and fusion group $\left(-0.4^{\circ}\right.$ to $\left.-11.1^{\circ}\right)$, whereas it was maintained in the control group $\left(-12.0^{\circ}\right.$ to $\left.-11.6^{\circ}\right)$ and had worsened in the laminoplasty-only group $\left(-1.7^{\circ}\right.$ to $\left.0.5^{\circ}, p<0.05\right)$ (Fig. 5). The authors concluded that improvement in patient outcomes after fusion was attributable to the improvement in cervical lordosis, improvement in posture, decompression of thickened posterior ligamentum flavum, and decompression of the area around the pre-surgical kyphotic deformity.

\section{Cervical spondylotic myelopathy and ossification of the posterior longitudinal ligament}

Segmental instability is an important pathological factor in CSM due to ossification of the posterior longitudinal ligament (OPLL). While laminoplasty has been widely used in CSM due to OPLL, progressive kyphosis and progressive ossified lesions on the follow-up remain as concerns, potentially leading to late neurological complications [15,16]. Chen et al. [12] reviewed a cohort of 15 patients with CSM due to OPLL who had dynamic instability and who were treated with unilateral open-door laminoplasty with titanium arch miniplate (no bone graft) and lateral mass screw fixation at unstable levels to provide immediate stabilization from 2005 to 2007 . Using this surgical technique, none of the 15 patients developed progressive kyphosis or progression of the ossified lesions in over 4 years of the follow-up. Furthermore, the JOA scores significantly improved at 6 months postoperatively and well-maintained at the follow-up (mean, $7.5 \pm 1.6$ points, preoperatively; $13.0 \pm 2.0$ points, 4 -years postoperatively) (Fig. 4). These patients experienced improvement in their neurological functions at a rate of $57.9 \%$ in addition to the improvement of neck pain at 4 -years postoperatively. These authors compared their results with another series 
of 83 patients with CSM due to OPLL treated with laminectomy and instrumented fusion [14]. They found nearly identical neurological recovery; however, laminectomy and instrumented fusion was associated with a higher risk of complications such as nerve root palsy and hematoma. Thus, the authors concluded that laminoplasty with fusion is safer than laminectomy with fusion, although they have similar clinical effectiveness [12].

\section{Cervical spondylotic myelopathy and traumatic cen- tral cord syndrome}

In acute subaxial traumatic central cord syndrome without fracture or dislocation, Song et al. [8] retrospectively reviewed the surgical management to evaluate the role of surgery in this patient population. Twenty-two patients were identified at a single institution from 1995 to 2002. Of the 22 patients presented, four received laminoplasty and fusion with lateral mass screws. This approach was used for patients with cervical spinal instability on dynamic radiographs and multilevel CSM or OPLL. The remainder of the patients received anterior decompression and fusion or laminoplasty alone. All of the patients who received laminoplasty and fusion presented after a fall (as opposed to a motor vehicle collision) and had pre-existing developmental canal stenosis. The interval between injury and surgery was 3 days for two patients, 6 days for one patient, and 37 days for another patient due to the compromise of pulmonary function associated with the injury. All of these patients improved neurologically by at least one FG at discharge (Fig. 3). Laminoplasty with lateral mass screw fusion provided rapid decompression and fixation with good results in patients, with unstable multilevel CSM or OPLL.

In the case series of Matsumura et al. [10] a 57-yearold construction worker with CSM, who fell from a 5-foot scaffolding, is described. Imaging demonstrated cervical canal stenosis from $\mathrm{C} 3$ to $\mathrm{C} 7$ with cord $\mathrm{T} 2$ hyperintense signal at both $\mathrm{C} 3-\mathrm{C} 4$ and $\mathrm{C} 6-\mathrm{C} 7$. In addition, a dynamic instability was noted between $\mathrm{C} 6$ and $\mathrm{C} 7$ in association with a fracture of the $\mathrm{C} 6$ facet joint extending into the lamina. The patient underwent posterolateral fixation from C5 to C7 with lateral mass plates and screws in addition to C3-C7 laminoplasty by the Kurokawa's method, involving insertion of hydroxyapatite spacers between the split lamina of $\mathrm{C} 3$ and $\mathrm{C} 4$ and the insertion of iliac bone graft secured with titanium wire between the lamina from
C5 to C7. This patient, who presented with moderate weakness, could ambulate independently at 1 month and achieved radiographic bony fusion at 15 months postoperatively.

\section{Cervical spondylotic myelopathy and athetoid cere- bral palsy}

Demura et al. [11] presented a retrospective case series at a single center on the management of CSM due to athetoid cerebral palsy using laminoplasty and pedicle screw fixation. Athetoid cerebral palsy is caused by perinatal cerebellar and basal ganglia damage and is characterized by involuntary movements of the neck, trunk, and extremities [16]. CSM occurs early in this patient population due to excessive mechanical stress caused by involuntary movements, which lead to cervical spine degeneration and instability. Patients with CSM, due to athetoid cerebral palsy, provide a unique treatment challenge because the involuntary neck movements increase the postoperative risk of kyphosis, pseudoarthrosis, and adjacent segment disease. A consecutive series of 15 patients underwent midline T-saw laminoplasty with spinous process splitting, followed by pedicle screw fixation and iliac bone grafts between the opened lamina and pedicle screws. Postoperatively, patients showed improvement of their sagittal alignment with $\mathrm{C} 2-\mathrm{C} 7$ angle improved from $11.0^{\circ} \pm 14.5^{\circ}$ of kyphosis to $1.5^{\circ} \pm 12.7^{\circ}$ (Fig. 5). The postoperative JOA scores had a mean improvement rate of $32 \%$ at the final follow-up (mean follow-up time, 71 months). There were two cases of adjacent segmental disease necessitating additional surgery; however, all patients achieved solid posterior bony fusion without needing rigid orthosis, and there were no neurovascular complications. This study concluded that pedicle screw fixation allowed for better biomechanical strength, while the laminoplasty opened a space for iliac bone grafting to facilitate fusion, which when combined, provided for strong stabilization. The patient demographic, associated pathology, procedure data, measure of neurological improvement, C2-C7 angle pre- and postoperatively, and follow-up from some previous studies are summarized in Table 1 [8-12].

\section{Study limitations}

This literature review aimed to explore the utility of laminoplasty in combination with instrumented fusion, and 
six articles, all of which were retrospective case series, were quantitatively analyzed. The present review was restricted to articles in the English literature; as such, some relevant articles may have been excluded. Retrospective reviews come with inherent bias and sources of error. Data were recruited by convenience sampling, such as performing chart reviews at our institution; thus, the results may not be representative of the general population and may be prone to selection bias. Meanwhile, confounding was also a possibility, as postoperative variables may have been distorted by the presence of other factors not considered during data collection. Moreover, as this was a retrospective study, causation was not determined, rather only associations were made. Rare scenarios such as CSM treated with both laminoplasty and posterior fusion require large sample sizes, whereas this retrospective institutional review was unlikely to yield this information. Lastly, wherever electronic records were utilized, potential bias was introduced from items copied and pasted, default tick boxes, and the use of boilerplate notes.

\section{Discussion}

The surgical management of CSM has diversified over the past 50 years. Previously, cervical laminectomy alone was the gold standard treatment for wide decompression at multiple levels. However, postoperative complications such as segmental instability, kyphotic deformity, compressive perineural adhesions, and late neurological deterioration necessitated the development of new techniques [5]. For avoidance of these complications, ventral decompression and fusion, laminoplasty, and posterior fusion in combination with either laminectomy or laminoplasty are techniques that have been developed and modified. In 2016, Tetreault et al. [17] reviewed 60 studies to assess the predictors of complications among different surgical approaches. While older age, longer operative time, and two-stage surgeries reflected increased risk, the authors found that surgical approach does not affect the rate of complications [17]. Meanwhile, over the past decade, neurosurgery as a profession has attempted to achieve consensus on the most effective surgical intervention for the treatment of multilevel CSM $[18,19]$.

However, there is no consensus regarding which surgical approach is superior in terms of safety and effectiveness across all patients with CSM. The goal of all of these surgical approaches-anterior cervical decompression and fusion, laminectomy, laminectomy and fusion, laminoplasty, and laminoplasty and fusion-is to decompress the spinal cord and maintain sagittal alignment to improve neurological function and stability. The determination of which approach will achieve this goal depends on the pathology specific to that individual. Often, a combination of these approaches is necessary. Several comparison studies are limited by their failure to group patients by pathoanatomical variations, leading to similar outcomes across all patients. Anterior cervical decompression and fusion affords only mild corrections of the local kyphosis [9]. Conventionally, laminoplasty has not been considered in patients with CSM who have kyphotic deformities because laminoplasty alone adequately achieves the goal of spinal cord decompression, but does not address sagittal alignment. Without fusion, these patients run a higher likelihood of postoperative kyphosis when compared with that by anterior approaches.

In 2016, Farrokhi et al. [20] outlined an algorithm for surgically approaching CSM based specifically on cervical sagittal imbalance and affected levels. The authors provided a well-illustrated stepwise surgical approach with suggested procedures including anterior cervical discectomy and fusion, or arthroplasty, laminoplasty, laminectomy, and posterior decompression with fusion. In this algorithm, the underlying pathology of the individual is not considered as a variable for determining the appropriate surgical approach. Moreover, the combination of laminoplasty with posterior instrumented fusion is not included as a proposed option.

In a systematic review in 2013, Lawrence et al. [6] identified eight level III retrospective cohort studies to compare the effectiveness and safety of anterior multilevel cervical discectomy or corpectomy and fusion with posterior cervical laminoplasty or laminectomy and fusion. Lawrence et al. [6] concluded that multilevel CSM management must consider whether the pathology is ventral or dorsal, focal or diffuse, stable or unstable, kyphotic or lordotic, and whether a radiculopathic component is present or absent. Data analysis revealed no clear effectiveness or safety advantage associated with either the anterior or posterior approach.

In 2003, Suda et al. [21] retrospectively analyzed cervical alignment in 114 patients with CSM before and after laminoplasty. The average preoperative cervical alignment (C2-C7) among these patients was $17.3^{\circ}$ of lordosis, with a range from $10^{\circ}$ kyphosis to $53^{\circ}$ lordosis. Twenty-three 
patients showed preoperative local kyphosis exceeding $5^{\circ}$. The average postoperative cervical alignment among these patients was $15.9^{\circ}$ of lordosis, with a range from $25^{\circ} \mathrm{ky}$ phosis to $49^{\circ}$ lordosis. Twenty patients showed preserved cervical lordosis. Preoperative MRI scans demonstrated cord signal intensity changes in 49 patients, which was associated with poor neurological recovery despite sufficient decompression with laminoplasty. Overall, the authors found that change in the MRI signal intensity and local kyphosis were the most significant prognostic factors for determining the neurological outcomes. The highest risk of poor neurological recovery was local kyphosis exceeding $13^{\circ}$, which was calculated with a logistic regression model [21].

Laminoplasty studies from over a decade ago indicated consistent development of postoperative kyphotic deformity and worsening cervical alignment, raising questions about the utility of this approach due to concern for poor prognosis. Although prior literature reviews found worsening alignment in $23 \%-50 \%$ of the reported cases, the mean cervical angle remained globally stable. Therefore, the known association between preoperative local kyphosis and worsened neurological prognosis is probably the result of pre-existing neurological damage, which could lead to further kyphotic deformity development. Comparison data on preoperative and postoperative cervical alignment in the literature is largely insufficient, with only $20 \%$ of laminoplasty studies reporting statistics. Furthermore, a recent literature review concluded that laminoplasty leads to favorable neurological outcomes and decreased complication rate; however, the change in the cervical alignment remains unclear [11].

In a meta-analysis of 10 non-randomized-controlled studies ( $\mathrm{n}=819$ patients) with CSM due to OPLL, Chen et al. [22] compared the effectiveness and safety of anterior corpectomy and fusion with that of laminoplasty. They found better neurological recovery and superior recovery rate in patients who underwent anterior corpectomy and fusion and concluded that an anterior approach is preferable in patients with kyphotic cervical alignment or central stenosis of $>60 \%$, while laminoplasty is safe and effective with adequate cervical lordosis. However, laminoplasty was performed less often in patients with preoperative kyphotic alignment due to reports of lower recovery rates and postoperative loss of cervical lordosis or kyphotic deformity development. Thus, the degree of local kyphosis was neither characterized nor compared for the determi- nation of the surgical approach [22].

The current literature is limited in its exploration of how reduction and stabilization with posterior instrumentation can be utilized in combination with laminoplasty for patients with multilevel CSM who have some level of kyphotic deformity and/or instability. Based on our review, laminoplasty with fusion can be considered for patients with multilevel CSM in a setting of local kyphotic deformity or instability, multilevel OPLL with associated segmental instability, and the need for strong stabilization in a setting of movement disorders such as athetoid cerebral palsy.

There are several particular advantages of this technique over the commonly practiced technique of laminectomy and fusion. First, having posterior elements available to serve as a fusion surface may increase the arthrodesis rates compared with laminectomy and fusion, where only the lateral masses are available to serve as a fusion surface. Second, this technique can be used to minimize the biomechanical tendency toward further kyphosis, which can be created with the removal of posterior tension band similar to that with laminectomy alone in patients that are anyway going to require fusion. Third, laminoplasty and fusion provides a protective bony layer not afforded by laminectomy and fusion and thus could theoretically reduce delayed post-surgical compression related to adhesions or scar over the open dural surface. Lastly, according to Chen et al. laminectomy and instrumented fusion was associated with a higher risk of complications such as nerve root palsy and hematoma compared with laminoplasty and fusion, which could also be attributable to previous listed advantage of bony protection over the dural surface [22].

Relatively few complications or disadvantages have been reported by those who performed cervical laminoplasty and concomitant posterior fusion. Chen et al. [22] reviewed a series of 83 patients with multilevel OPLL treated with laminoplasty and instrumented fusion and found only one with C5 palsy, which completely recovered 2 months after the surgery. None of the patients developed progressive kyphosis or significant progression of the ossified lesions. Matsumura et al. [10] described two cases of patients with cervical canal stenosis who underwent laminoplasty with interspinous longitudinal iliac bone graft in combination with plate fixation. They noted that the operative time and blood loss was "slightly greater than the usual laminoplasty method or lateral mass plat- 
ing," but also reported that "no serious postoperative complication" occurred. Uehara et al. [7] reviewed 13 cases and noted "no neurovascular complications", which was echoed by Demura et al. [11], who reviewed 17 cases at a single institution. Miyamoto et al. [9] described 30 cases and reported no requirement of additional surgery due to complications.

\section{Conclusions}

Cervical laminoplasty with fusion can be considered for patients with CSM in a setting of local kyphotic deformity, OPLL-associated segmental instability, and the need for strong stabilization in the setting of movement disorders such as athetoid cerebral palsy. This approach could also be considered in young patients with a full range of flexion and extension motion and who are at risk of swan neck deformity after laminectomy. The performance of laminoplasty for the preservation of the posterior elements with concomitant fusion may provide benefit in this as well as in other unexplored scenarios and should therefore be considered.

\section{Conflict of Interest}

No potential conflict of interest relevant to this article was reported.

\section{Acknowledgments}

The authors thank Shirley McCartney, PhD, for editorial assistance.

\section{References}

1. Wang MC, Kreuter W, Wolfla CE, Maiman DJ, Deyo RA. Trends and variations in cervical spine surgery in the United States: Medicare beneficiaries, 1992 to 2005. Spine (Phila Pa 1976) 2009;34:955-61.

2. Seng C, Tow BP, Siddiqui MA, et al. Surgically treated cervical myelopathy: a functional outcome comparison study between multilevel anterior cervical decompression fusion with instrumentation and posterior laminoplasty. Spine J 2013;13:723-31.

3. Dadashev VY, Rodts Jr GE. Treatment of disk and ligamentous diseases of the cervical spine. In: Winn HR, editor. Youmans neurological surgery. 6th ed.
Philadelphia (PA): Elsevier/Saunders; 2011. p.285967.

4. Jandial R, Garfin SR. Best evidence for spine surgery: 20 cardinal cases. Philadelphia (PA): Elsevier/Saunders; 2012.

5. Highsmith JM, Dhall SS, Haid RW Jr, Rodts GE Jr, Mummaneni PV. Treatment of cervical stenotic myelopathy: a cost and outcome comparison of laminoplasty versus laminectomy and lateral mass fusion. J Neurosurg Spine 2011;14:619-25.

6. Lawrence BD, Jacobs WB, Norvell DC, Hermsmeyer JT, Chapman JR, Brodke DS. Anterior versus posterior approach for treatment of cervical spondylotic myelopathy: a systematic review. Spine (Phila $\mathrm{Pa}$ 1976) 2013;38(22 Suppl 1):S173-82.

7. Uehara M, Takahashi J, Ogihara N, et al. Cervical pedicle screw fixation combined with laminoplasty for cervical spondylotic myelopathy with instability. Asian Spine J 2012;6:241-8.

8. Song J, Mizuno J, Nakagawa H, Inoue T. Surgery for acute subaxial traumatic central cord syndrome without fracture or dislocation. J Clin Neurosci 2005;12:438-43.

9. Miyamoto H, Maeno K, Uno K, Kakutani K, Nishida K, Sumi M. Outcomes of surgical intervention for cervical spondylotic myelopathy accompanying local kyphosis (comparison between laminoplasty alone and posterior reconstruction surgery using the screw-rod system). Eur Spine J 2014;23:341-6.

10. Matsumura A, Yanaka K, Akutsu H, Noguchi S, Moritake T, Nose T. Combined laminoplasty with posterior lateral mass plate for unstable spondylotic cervical canal stenosis: technical note. Neurol Med Chir (Tokyo) 2003;43:514-9.

11. Demura S, Murakami H, Kawahara N, Kato S, Yoshioka K, Tsuchiya H. Laminoplasty and pedicle screw fixation for cervical myelopathy associated with athetoid cerebral palsy: minimum 5-year follow-up. Spine (Phila Pa 1976) 2013;38:1764-9.

12. Chen Y, Wang X, Chen D, Miao J, Liao X, Yu F. Posterior hybrid technique for ossification of the posterior longitudinal ligament associated with segmental instability in the cervical spine. J Spinal Disord Tech 2014;27:240-4.

13. Shikata J, Yamamuro T, Shimizu K, Saito T. Combined laminoplasty and posterolateral fusion for spinal canal surgery in children and adolescents. Clin 
Orthop Relat Res 1990;(259):92-9.

14. Raimondi AJ, Gutierrez FA, Di Rocco C. Laminotomy and total reconstruction of the posterior spinal arch for spinal canal surgery in childhood. J Neurosurg 1976;45:555-60.

15. Iwasaki M, Kawaguchi Y, Kimura T, Yonenobu K. Long-term results of expansive laminoplasty for ossification of the posterior longitudinal ligament of the cervical spine: more than 10 years follow up. J Neurosurg 2002 Mar;96(2 Suppl):180-9.

16. Ogawa Y, Chiba K, Matsumoto M, et al. Long-term results after expansive open-door laminoplasty for the segmental-type of ossification of the posterior longitudinal ligament of the cervical spine: a comparison with nonsegmental-type lesions. J Neurosurg Spine 2005;3:198-204.

17. Tetreault L, Ibrahim A, Cote P, Singh A, Fehlings MG. A systematic review of clinical and surgical predictors of complications following surgery for degenerative cervical myelopathy. J Neurosurg Spine 2016;24:77-99.

18. Fehlings MG, Smith JS, Kopjar B, et al. Perioperative and delayed complications associated with the surgical treatment of cervical spondylotic myelopathy based on 302 patients from the AOSpine North America Cervical Spondylotic Myelopathy Study. J Neurosurg Spine 2012;16:425-32.

19. Shamji MF, Cook C, Tackett S, Brown C, Isaacs RE. Impact of preoperative neurological status on perioperative morbidity associated with anterior and posterior cervical fusion. J Neurosurg Spine 2008;9:10-6.

20. Farrokhi MR, Ghaffarpasand F, Khani M, Gholami M. An evidence-based stepwise surgical approach to cervical spondylotic myelopathy: a narrative review of the current literature. World Neurosurg 2016;94:97110.

21. Suda K, Abumi K, Ito M, Shono Y, Kaneda K, Fujiya M. Local kyphosis reduces surgical outcomes of expansive open-door laminoplasty for cervical spondylotic myelopathy. Spine (Phila Pa 1976) 2003;28:125862.

22. Chen Z, Liu B, Dong J, et al. Comparison of anterior corpectomy and fusion versus laminoplasty for the treatment of cervical ossification of posterior longitudinal ligament: a meta-analysis. Neurosurg Focus 2016;40:E8. 